\title{
Propuesta de materiales reciclables para la fabricación de placas vehiculares en la República de Panamá
}

\section{Proposal of recyclable materials for the manufacture of license plates in the Republic of Panama}

\author{
María Serrano ${ }^{l}$, Alexander Jaén ${ }^{l}$, Nacarí Marín ${ }^{2 *}$ \\ ${ }^{1}$ Licenciatura en Ingeniería Electromecánica, Centro Regional de Azuero, Universidad Tecnológica de Panamá, ${ }^{2}$ Facultad de \\ Ingeniería Mecánica, Centro Regional de Azuero, Universidad Tecnológica de Panamá.
}

\begin{abstract}
Resumen Con base al último registro realizado por el Instituto Nacional de Estadística y Censo (INEC) en el año 2018, se registraron 1847547 vehículos, los cuales deben tener sus placas en regla para poder circular libremente. En Panamá, la renovación de las placas vehiculares es realizada cada año. Anteriormente, no se tenía un control absoluto sobre las placas de años pasados, con una cantidad exorbitante de aluminio desperdiciado por año. Se le suma la producción de basura con un aproximado de 2.00 toneladas diarias, que en conjunto aumenta la contaminación ambiental. El objetivo principal de este artículo es presentar una propuesta para el aprovechamiento de materiales reciclables en la fabricación de placas vehiculares en la República de Panamá. En este documento se evalúan las propiedades mecánicas de dos materiales: aluminio reciclado y rPET, para llegar a la conclusión que, con la implementación de distintos procesos para su adecuación en láminas, pueden ser fácilmente utilizables para el proceso, reduciendo así la cantidad de desechos que se posee.
\end{abstract}

Palabras clave Aluminio, ambiente, contaminación, PET, placas, reciclaje, vehículos.

\begin{abstract}
Based on the latest registration carried out by the National Institute of Statistics and Census (INEC) in 2018, 1,847,547 vehicles were registered, which must have their license plates in order to be able to circulate freely. In Panama, the renewal of vehicle plates is carried out every year, however, there is no absolute control over the plates of previous years, thus having an exorbitant amount of aluminum wasted per year. To this is added the production of garbage with an approximate of 2.00 tons per day, which together increases environmental pollution. The main objective of this article is to present a proposal for the use of recyclable materials in the manufacture of license plates in the Republic of Panama. In this document, the mechanical properties of two materials are evaluated: recycled aluminum and rPET, reaching the conclusion that with the implementation of different processes for their adaptation in sheets, they can be easily usable for the process, thus reducing the amount of waste that is has.
\end{abstract}

Keywords Aluminum, environment, pollution, PET, plates, recycling, vehicles.

* Corresponding author: nacari.marin@utp.ac.pa

\section{Introducción}

Se calcula que en Panamá son producidas aproximadamente 2.00 toneladas de basura al día, de las que un $60 \%$ se encuentra constituida por plásticos, latas, entre otros materiales o elementos con potencial para ser reciclados.

La contaminación, está afectando no solo a Panamá, sino a todas las partes del mundo. Hasta el 2018, existían más de un millón de automóviles registrados en la Autoridad de Tránsito y Transporte Terrestre (ATTT) de Panamá. Esta cantidad se atribuye al crecimiento de la población y a las deficiencias en el transporte público [1].

Cada automóvil debe contar con una placa de registro vehicular, para poder circular por las vías terrestres del país y se confeccionan en la Escuela Vocacional de Chapala, Panamá.
Se ha comprobado que reciclar tiene un sin número de beneficios, como: La reducción de la contaminación por desechos o desperdicios y la conservación de la vida del medioambiente. Con base en esta premisa, se propone un proyecto que busca concientizar a la población sobre el tema de reutilización de materiales con potencial para ser reciclados, en la confección de las placas vehiculares.

Actualmente, se considera el estudio del reciclaje en las placas vehiculares, que deben ser actualizadas cada año, lo que representa un gasto, tanto de factor monetario como en la materia prima que queda, contribuyendo al incremento de los desechos municipales y en la contaminación ambiental.

El objetivo de este estudio es realizar una revisión del estado del arte que permita plantear las ventajas y desventajas 
de la utilización de materiales reciclados, en la fabricación de placas vehiculares.

\section{Antecedentes}

En base a los datos más actualizados del Instituto Nacional de Estadística y Censo en Panamá (INEC), en el año 2018, se registraron 953261 vehículos, los que incluyen automóviles, bicicletas y motocicletas y 894286 autos comerciales [2]. Aunque es una cantidad bastante elevada, existen indicios en la disminución de la compra de vehículos cero kilómetros en un $15 \%$ durante los tres últimos años [3]; la compra de vehículos de segunda mano ha ido en aumento.

Todos estos vehículos y los que se adquirieron en el periodo 2019-2020, requieren de la actualización de su placa cada año, lo que conlleva para el estado un gasto en materia prima y en su confección.

Los encargados de confeccionar las placas son los miembros de la Escuela Vocacional de Chapala, ubicada en Arraiján quienes cobran 5.00 balboas por cada placa realizada. En el año 2019, 3.5 millones de balboas fueron destinados a la fabricación de placas, solo en el Municipio de Panamá [4] [5].

Una forma útil de reducir los costos y ayudar al ambiente es considerar la utilización de materiales reciclables, en este caso, para la confección de placas vehiculares.

La figura 1, muestra la placa vehicular correspondiente al año 2020, a modo de ilustración.

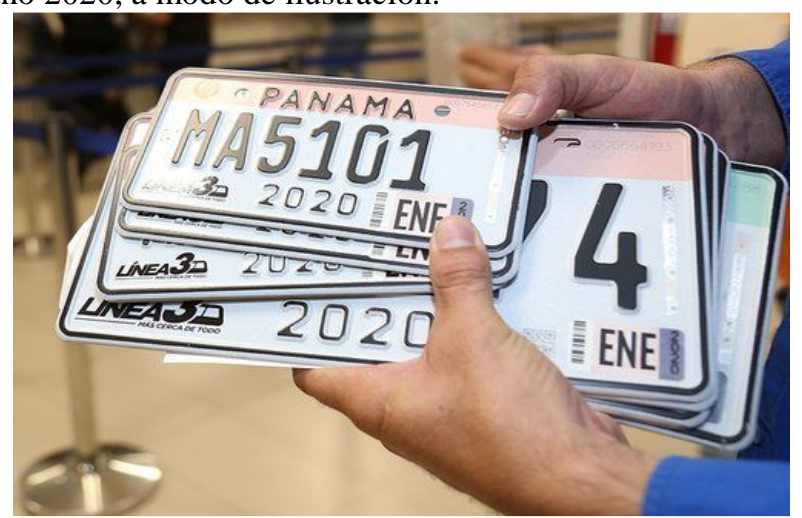

Figura 1. Placas vehiculares de Panamá.

Fuente: Telemetro.com [6].

El pasado 7 de octubre del 2020, se aprobó en tercer debate, el Proyecto de Ley 434, el cual establece el uso de la placa vehicular por un periodo de 5 años. Con este proyecto se estima que se ayude a mitigar el daño ecológico causado por el aluminio calibre 24 que se utiliza en el proceso de fabricación de las placas [7]. Esta iniciativa de ley representa sin duda alguna, una disminución en la contaminación y si se evalúa la utilización de materiales reciclables los beneficios serían aún mayores.

\section{Metodología}

En esta sección se detallan las distintas consideraciones tomadas en cuenta para el desarrollo de esta revisión literaria.

\subsection{Planteamiento de la problemática}

Según los datos de [8] solo el 5\% de los desechos que se vierten en Panamá son reciclados, lo que lleva a la conclusión que la población del país no sabe reciclar, que puede ser una de las mayores limitantes de la propuesta que se presentará en este estudio. No obstante, se basa en la investigación de materiales reciclados que pueden ser sometidos a procesos térmicos y mecánicos y que aun así sigan manteniendo sus propiedades.

Es importante destacar que las placas vehiculares cuentan con especificaciones de alta seguridad y cumplen ciertas normas que deben ser consideradas. Algunas de estas normas se detallan en la figura 2 [9]:

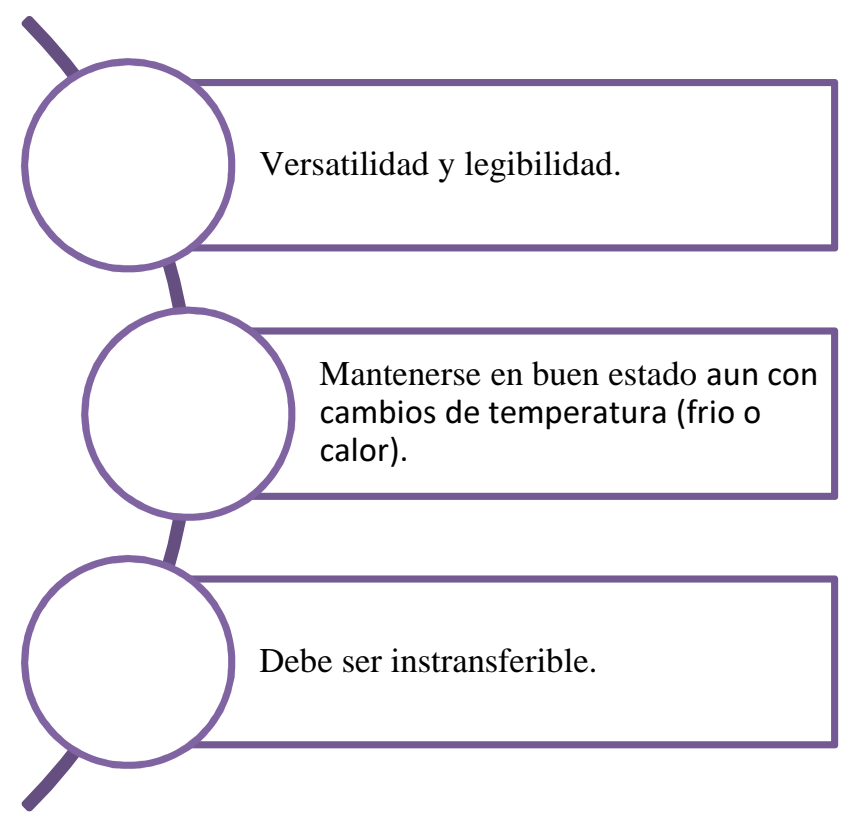

Figura 2. Especificaciones que deben cumplir las placas vehiculares en la República de Panamá.

Las distintas especificaciones que se muestran en la figura 2 son la base para la obtención de los materiales que pueden ser utilizables para tales fines.

A continuación, se presenta información sobre los distintos materiales que son propuestos como una alternativa al uso de aluminio calibre 24 en las placas vehiculares seguido de su proceso de reciclaje y finalmente la propuesta de utilización.

\subsection{Evaluación de materiales reciclables}

Existen diversos tipos de materiales que pueden ser reciclados, pero no todos pueden ser utilizados para realizar las placas vehiculares. A continuación, presentamos una lista de 
los materiales más resistentes y óptimos para tal fin, identificados a través de la revisión bibliográfica consultada.

\subsubsection{Aluminio}

El aluminio es considerado parte de los "metales ligeros", caracterizados por tener una baja densidad y por sustituir a materiales como el acero en estructuras y componentes, debido a su bajo peso [10].

Ahora bien, las latas de aluminio tardan aproximadamente diez años en degradarse y, su utilización en forma masiva provoca problemas de diversa índole [11]. Según los autores de [12] solo el $42 \%$ de los envases de lata provenientes de escuelas y domicilios son reciclados.

En la actualidad, el aluminio es utilizado para la elaboración de las placas vehiculares, pues es un material resistente y anticorrosivo, cualidades necesarias, que continuamente están expuestas a cambios bruscos de temperatura y humedad.

El aluminio reciclado es uno de los materiales más utilizados. En [13] se presentan propuestas de reciclado de latas de aluminio y se concluye considerando que el aluminio reciclado mantiene propiedades mecánicas similares al aluminio virgen.

Por otra parte, el escrito presentado por [14], se revela que el 95\% del aluminio recuperado únicamente en latas de bebidas es utilizado para la confección de láminas de aluminio, de las cuales resultan nuevas latas, con un proceso que es realizado fuera de país.

\subsubsection{PET}

Los plásticos se diferencian de otros materiales por su relación entre resistencia y calidad, que poseen cualidades para un buen aislamiento térmico y eléctrico [15].

Por su parte, el polietileno de tereftalato (PET) es considerado un polímero de condensación termoplástico, utilizado en la producción de envases de bebidas como refrescos y fibras textiles. Está constituido por petróleo crudo, gas y aire. [15]

Las bolsas de plástico tardan aproximadamente 150 años en degradarse completamente, mientras que las botellas, platos y vasos de plástico tardan 450 años y 50 años respectivamente [16].

Desde el 20 de julio del año 2019, se implementó la prohibición del uso de bolsas de plástico para el transporte de productos y mercancías; aún se siguen fabricando innumerables productos a base de este material.

El tereftalato de polietileno o PET, es otra propuesta a considerar por la gran demanda que existe sobre las botellas hechas a base de PET, poniendo su reciclaje como un punto a favor. En la actualidad se ha buscado el reciclaje de este material y se ha visto en aplicaciones como material para impresoras 3D, confección de envases, uso en zapatillas, como refuerzo en construcciones, entre otros [17].
Se ha comprobado que el rPET (tereftalato de polietileno reciclado) se puede reactivar para conseguir las mismas funcionalidades del PET virgen, mejorando así algunas propiedades como: Resistencia al impacto, brillo, color, entre otras [18].

En [19] se presentan los procesos que se requieren para el reciclaje del PET, los que permiten adecuarlo para su utilización. Y se propone un sistema de reciclaje de este material, como punto clave para reducir la cantidad de desechos existente.

Luego se proponer los materiales anteriores, se expondrá el proceso de reciclaje para cada uno, incluyendo los tratamientos que debe recibir para convertirse en una materia prima utilizable.

\subsection{Proceso de reciclaje del aluminio}

El aluminio reciclado mantiene las propiedades iniciales, variando en aproximadamente un 5\%. En [10] se indica que el material puede reciclarse varias veces, pero se logra mediante una serie de procesos que permitan trabajarlo de manera eficiente, para obtener las propiedades requeridas. Un ejemplo de cómo se da este proceso es mediante el reciclaje de latas de refrescos, cuyo desarrollo se muestra a continuación [10]:

- Recolectar los desechos metálicos y clasificarlos, pues varía dependiendo de su aplicación.

- Posterior a su separación, este pasa a un proceso de rectificación donde se evalúa nuevamente el material para que no se encuentren impurezas o materiales no deseables como por ejemplo otros metales.

- Ulteriormente, se compactan las latas sometiéndolas a presión para aplastarlas creando pacas.

- En este punto se descompactan y se introducen al horno para fundirse y luego crear bloques de aluminio.

- En este punto, en el caso de las latas, se utiliza el proceso de laminado donde se disminuye el diámetro al tamaño requerido. El laminado permite disminuir el tamaño de los bloques y a su vez se le da un tratamiento en frío, para mejorar la resistencia de los elementos.

Basados en este caso, la forma de replicar este sistema de reciclado, permitiría la manufactura de la materia prima y a su vez la reducción de las latas como desechos. Los autores de [9] detallan que una forma de mejorar las propiedades del aluminio reciclado es añadiendo un pequeño porcentaje de silicio en el proceso de fundición para generar propiedades de moldeo, soldabilidad y resistencia a la corrosión [20].

\subsection{Proceso de reciclaje del PET}

En [21] se describen dos métodos para realizar el proceso de reciclaje del PET, los que dependen de la utilidad que vaya a recibir el material después de ser reciclado. De esta forma, se cuenta con una opción de proceso de reciclaje, en donde el material se transforma sin necesidad que sea limpiado y otro 
en el cual el material es pasado por un proceso de limpieza en donde se eliminan bacterias y factores que no sean propios del material. [21]:

Seguidamente, detallamos los procesos presentados en

\subsubsection{Reciclaje químico}

Este proceso se basa en elevar la temperatura del polímero hasta llegar a separar las partículas. Este polímero previamente reciclado es sometido a un proceso de descomposición hasta que sus partículas sean transformadas en monómeros (molécula simple) y así puedan ser transformados en nuevos polímeros que son utilizados en industrias.

\subsubsection{Reciclaje mecánico}

Es el método más común, el cual se basa en triturar el plástico en pequeños trozos para posteriormente colocarlo en una máquina extrusora, que le dará una mejora a sus propiedades y a la vez lo moldeará según la forma deseada, en este caso una lámina lo suficientemente resistente.

Cabe destacar, que este proceso es realizado en los polímeros que se utilizan para envasar productos de consumo y posee ciertas condiciones que deben cumplirse, entre ellas:

- Los plásticos utilizados deben ser homogéneos, es decir, que deben pasar por un proceso de clasificación previo a la trituración.

- No deben contener ninguna partícula externa al material base, ya que podría dañar la extrusora.

En [15] se incluye, en la clasificación sobre los tipos de reciclaje para el PET, el reciclaje energético. Este reciclaje es utilizado para producir energía calorífica.

El proceso que conlleva recolectar el plástico para su adecuación será la clave para poder llegar a utilizarlo de una manera que beneficie al medio ambiente.

\section{Propuesta de fabricación de láminas para utilización en placas vehiculares}

Una vez analizados los procesos para el reciclaje de ambos materiales se procederá a mostrar la propuesta desarrollada para el proceso de fabricación de las láminas.

\subsection{Aluminio}

La tabla 1, muestra el proceso que se propone para la realización de las láminas de aluminio reciclado basado en un desarrollo mecánico.
Tabla 1. Proceso propuesto para la fabricación de láminas para la utilización en placas vehiculares

\begin{tabular}{|c|c|}
\hline $\begin{array}{c}\text { Nombre del } \\
\text { proceso }\end{array}$ & Descripción del proceso \\
\hline Recolección & $\begin{array}{l}\text { El proceso de reciclaje de } \\
\text { materiales comienza con la } \\
\text { recolección de latas } \\
\text { utilizadas en buen estado y } \\
\text { limpias, que garantizará el } \\
\text { cumplimiento de los } \\
\text { objetivos. }\end{array}$ \\
\hline Clasificación & $\begin{array}{l}\text { Las latas deben ser } \\
\text { clasificadas verificando que } \\
\text { no existan residuos de otros } \\
\text { elementos en ellas. }\end{array}$ \\
\hline Compactación & $\begin{array}{l}\text { El proceso de compactación } \\
\text { consiste en la formación de } \\
\text { grandes bloques para obtener } \\
\text { un mejor manejo. }\end{array}$ \\
\hline $\begin{array}{l}\text { Fundición: } \\
\text { Formación de } \\
\text { bloques }\end{array}$ & $\begin{array}{l}\text { El proceso de fundición } \\
\text { consiste en llenar un molde } \\
\text { con la cantidad de aluminio } \\
\text { fundido requerido con la } \\
\text { finalidad de extraer, una vez } \\
\text { el líquido haya solidificado, } \\
\text { una pieza con la medida y } \\
\text { forma del molde utilizado } \\
\text { [22]. El autor de [23] estudia } \\
\text { mezclas de modelo óptimas } \\
\text { para fundición de aluminio } \\
\text { considerando distintos } \\
\text { parámetros. }\end{array}$ \\
\hline $\begin{array}{c}\text { Laminación de } \\
\text { los bloques }\end{array}$ & $\begin{array}{l}\text { La laminación consiste en la } \\
\text { producción de una } \\
\text { deformación permanente en } \\
\text { el material, aprovechando su } \\
\text { ductilidad [24]. Una vez que } \\
\text { se haya realizado la } \\
\text { formación de bloques, estos } \\
\text { pasarán por un proceso de } \\
\text { laminado en donde se } \\
\text { reducirá su espesor y a su vez } \\
\text { se mejorarán sus propiedades } \\
\text { mecánicas, entregándose la } \\
\text { materia prima para el proceso } \\
\text { de fabricación de las placas } \\
\text { vehiculares. }\end{array}$ \\
\hline \multicolumn{2}{|c|}{ Creación de placas vehiculares } \\
\hline
\end{tabular}


Inmediatamente de realizar todo el proceso mostrado en la tabla 1, se debe disponer de láminas con el mismo espesor y proporción de las utilizadas para la fabricación de las placas vehiculares.

\subsection{PET}

La figura 3, detalla el diagrama de flujo del proceso propuesto para la realización de láminas a base de tereftalato de polietileno reciclado [25].

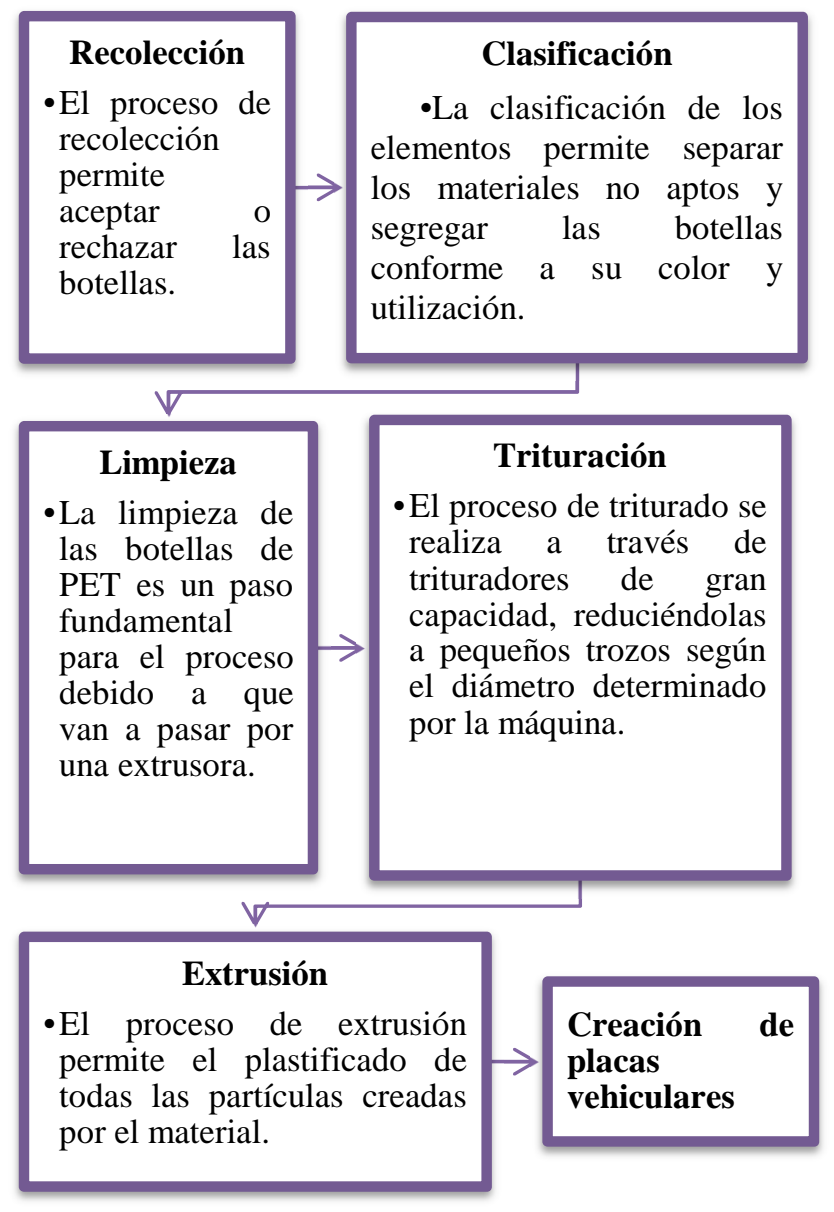

Figura 3. Diagrama de flujo de la propuesta de fabricación de láminas a base de PET.

A diferencia del proceso planteado para el aluminio (ver tabla 1) la figura 3, muestra diferentes pasos que deben seguirse, como: El proceso de limpieza puede realizarse antes o después de la trituración de las botellas; un punto importante a tratar cuando consideramos la utilización de botellas de plástico recicladas es la coloración debido a que la mayoría de las botellas de PET son transparentes. Este proceso se realiza junto con la extrusión y se logra con la utilización de distintos pigmentos y master o colorantes, los cuales son seleccionados dependiendo del tipo de características necesarias en el producto final [25].

\section{Trabajos futuros}

A futuro se plantea la realización de un prototipo de las placas vehiculares, con la utilización de los materiales estudiados en este artículo, aplicando los procesos de reciclaje existentes y exponiéndolas a las condiciones sujetas para su transformación a láminas. De igual manera, este prototipo tendrá características distintas, así como las referidas en este documento con respecto al diseño de las placas vehiculares que no puede ser transferible.

Pretendemos que el material reciclado sea proveniente de los hogares y comercios panameños; para lograrlo debemos concientizar a la población sobre el correcto uso que debemos darles a los desechos.

\section{Conclusiones}

La contaminación va en aumento en todo el mundo y Panamá, no es la excepción. Para poder disminuirla debemos buscar alternativas que contemplen la reutilización de los desechos. A continuación, presentamos las conclusiones obtenidas con el desarrollo del estudio:

- Los materiales como el PET y el aluminio proveniente de las botellas y latas de bebidas fueron las opciones estudiadas que poseen una gran demanda en el mercado, con mayor materia prima.

- El impacto que puede tener la elaboración de placas vehiculares a base de materiales reciclables deberá ser favorable en la reducción de desechos y por ende en la reducción de la contaminación.

- Con la cantidad de vehículos en circulación por el país actualmente, como una de las ventajas de esta propuesta es la minimización los gastos en láminas de aluminio de primera mano utilizadas para la confección de las placas vehiculares, que por el momento deben ser modificadas cada año.

- De acuerdo a los estudios citados en este artículo, se revela que tanto el aluminio, como el PET mantienen sus propiedades mecánicas, aún después de ser reciclados.

- Para poder obtener resultados favorables, se deben seguir correctamente los procesos para el reciclaje de ambos materiales debido a que esto garantizará que sus propiedades perduren.

\section{REFERENCIAS}

[1] Redacción Digital La Estrella, «Incorporarán letras a las placas vehiculares,» La Estrella de Panamá, Panamá, 2011.

[2] Instituto Nacional de Estadística y Censo - Panamá, 17 Octubre 2020. [En línea]. Available: 
https://www.inec.gob.pa/archivos/P0705547520200113125921C uadro\%201.pdf.

[3] I. Gordón, «Sector automotriz, con caída del $57 \%$ en el primer semestre intentará encender motores,» La estrella de Panamá, 16 Agosto 2020.

[4] G. Pineda, «Ejecutivo realizó compra directa para aluminio de las placas de enero 2020,» Mi Diario, Panamá, 2020.

[5] J. Simmons, «Más de dos mil jóvenes en riesgo social ha graduado la Escuela Vocacional de Chapala,» Editora Panamá América S.A, Panamá, 2019.

[6] N. Gónzalez, «Alcaldía de Panamá ofrece servicio de entrega de placas a domicilio,» Panamá, 2020.

[7] G. Aparicio, «Aprueban en tercer debate proyecto de ley que dará vigencia de cinco años a la placa vehicular,» La Estrella de Panamá, Panamá, 2020.

[8] J. Torrijos Legazpi, «¿Es el reciclaje la solución a todo el problema ambiental?,» Panamá América , Panamá, 2019.

[9] Autoridad de Transito y Transporte Terrestre de Panamá, «Vehículos deben llevar la placa visible y en buen estado,» 8 Marzo 2016. [En línea]. Available: http://www.transito.gob.pa/noticia/vehiculos-deben-llevar-laplaca-visible-y-en-buen-estado. [Último acceso: 12 Marzo 2021].

[10] F. Millán Delgado, D. P. Sánchez García y J. J. Olaya Flórez, «Reciclaje de aluminio: oportunidades de desarrollo en Bogotá (Colombia),» Gestión y Ambiente, vol. 18, n 2, pp. 135-152, 2015.

[11] División de industria , Ciencias para metalmecánica, Servicio nacional de operaciones SENA.

[12] C. Martínez, L. Marín y X. Socorro, «Contaminación de basura,» Juigalpa, 2017.

[13] F. Millán Delgado, Fabricación y caracterización de la aleación de aluminio reciclado con adición de silicio particulado, Bogotá, 2016.

[14] A. Samaniego, «Panamá tiene mucha basura, pero vende pocos desechos,» La Estrella de Panamá, Panamá, 2020.

[15] J. J. Bolaños, «Reciclado de Plástico PET,» Diciembre 2019. [En línea].

Available: https://core.ac.uk/download/pdf/250640335.pdf. [Último acceso: 18 Octubre 2020].

[16] Aquae Fundación, «¿Cuál es el tiempo de degradación de los residuos inorgánicos?,» [En línea]. Available: https://www.fundacionaquae.org/cuanto-tiempo-tardandegradarse-desechos/. [Último acceso: 14 Marzo 2021].

[17] M. D, «Tecnología de los Plásticos,» 30 Mayo 2011. [En línea]. Available:

https://tecnologiadelosplasticos.blogspot.com/2011/05/pet.html\# : :text=Debido\%20a\%20su\%20resistencia\%2C\%20el,para\%20p inturas $\% 20 y \% 20$ cepillos\%20industriales.. [Último acceso: 14 Marzo 2021].

[18] A. Valera, F. Juanes, J. Miguez y M. Gónzalez, «Aleaciones de polipropileno (pp) y polietilentereftalato (pet) reciclados y estudios de sus propiedades,» Anales de mecánica de la fractura, vol. II, no 25, pp. 748-752, 2008.

[19] R. Rivera Távara, Propuesta de reciclaje mecánico de plásticos en la ciudad de Piura, Piura, 2004. -
[20] J. M. Bayo, «Propiedades de la Aleación Al-Si: Resistencia y Expansión Térmica,» 29 Julio 2009. [En línea]. Available: https://ingenieriademateriales.wordpress.com/2009/07/29/propie dades-de-la-aleacion-al-si-rsistencia-y-expansion-termica/.

[Último acceso: 14 Marzo 2021].

[21] C. Vargas y J. Cabezas, «Automatización de un proceso mecánico de reciclaje de bajo,» 2019. [En línea]. Available: https://repository.ucatolica.edu.co/bitstream/10983/23500/1/Trab ajo $\% 20 \mathrm{de} \% 20 \mathrm{Grado} \% 20 \% 28 \mathrm{Botellas} \% 20 \mathrm{PET} \% 29$.pdf. [Último acceso: 18 Octubre 2020].

[22] Recam Láser, «¿Qué es la fundición de aluminio?,» [En línea]. Available: https://recamlaser.com/es/que-es-la-fundicion-dealuminio/. [Último acceso: 14 Marzo 2021].

[23] F. Revelo, «Selección de la mezcla de moldeo óptima para fundición de aluminio con el uso de modelos gasificables,» Quito, 2011.

[24] J. Enríquez, E. Tremps, S. De Elío, Fernández y Daniel, «Laminación,» Madrid, 2010.

[25] La red de reciclados de plástico, «El proceso de reciclaje de plástico» [En línea]. Available: http://www.recicladoslared.es/proceso-de-reciclaje-de-plasticos/. [Último acceso: 15 Marzo 2021]. 